\title{
Analytical approach predicting water bidirectional transfers: application to micro and furrow irrigation
}

\author{
D. Crevoisier \\ Irrigation Research Unit, Cemagref Montpellier, France
}

\begin{abstract}
This paper presents an analytical or semi analytical method capable of predicting water bidirectional transfers in a soil under different irrigation systems, and specifically for micro and furrow irrigation systems. The method developed in this article uses the Green's function to solve Richards' equation. Some assumptions are made that allow the equation to be linearized and thus solved. The Green's function is a well-known method used to solve the partial differential equation (PDE) with constant coefficients in simple geometries and general boundary conditions. The singularity of the method lies in its approach to Richards' equation in real irrigation contexts as it superposes simple solutions which can be treated by Green's function method. This work has two main aims: to propose analytical and explicit forms of water content in the soil, and to treat irrigation scenarios (unspecified furrow shapes in the case of furrow irrigation, heterogeneous initial conditions, which take into account precipitation events and plant uptakes, etc.) in a simple and operational manner. It also allows the evaluation of the coefficients of the solute transfers equation which depend on soil water content. This equation can then be solved with the same approach developed for water transfers. We present here the main principles of the model, the first results and improvements that could be made in the future.

Keywords: furrow irrigation, analytical method, Green's function, water and solute transfers, bidirectional.
\end{abstract}

\section{Introduction}

Inadequate irrigation and fertilization practices can have important environmental impacts: waste of water, nitrate pollution. Furrow irrigation is one 
of the most commonly used irrigation system in the world and micro irrigation systems installations are on the increase. A better understanding of water and nitrate transfers specific to these irrigation systems and fertilization practices could reduce water drainage and nitrate leaching.

Unidirectional models combining water and solute transfers, soil chemical and plant uptake exist and are able to simulate changes occurring in the soil and plant state along a whole crop season $[2,6]$. The main fertilization practices concentrate nitrate in the top layers of the ridge in the context of furrow irrigation. Experiments shows that the nitrate distribution in the soil highly depends on irrigation practices: impact of the water application depth [7], use of an alternative furrow practice, which consists in irrigating every second furrows and applying fertilizer in dry furrows [9]. Here, lateral transfers increase due to the initial distribution of nitrate and unidirectional modelling isn't useful when predicting the fate of water and nitrate in the soil profile. This observation is all the more relevant under environmental contexts where water and nitrate application is adjusted to plants' needs.

Some numerical models can be used to predict water and solute bidirectional dynamics $[12,14]$, but require a large set of parameters and significant computing time. General analytical solutions concerning water transfers have been developed $[1,8]$, models specialized in drip irrigation have been adapted from general methods $[3,15]$ and other models concerning furrow irrigation allow the simulation of cumulative infiltration for a given opportunity time [11, 16]. But in the context of furrow irrigation, the prediction of nitrate leaching requires a better understanding of water and solutes bidirectional transfers' mechanisms.

The model developed in this work, predicts soil water transfers, analytically or semi analytically, under different irrigation scenarios. In the case of furrow irrigation, the resolution of the Richards' equation poses 4 major problems: the significant non-linearity of the Richards' equation, the complexity of the geometry, the mixed boundary conditions on the soil surface and the processing of heterogeneous initial conditions. This work proposes methods to solve each of these problems. The first section of this article deals with the linearization of the equation and its resolution in some theoretical cases. The second section explains how these simple solutions are superposed to recompose the initial complex problem solution. Lastly, improvements of the model and its adaptation to solute transfers, plant uptakes and atmospheric conditions are presented.

\section{Resolution of the transfer equation in theoretical cases}

The theoretical cases treated in this section concern sloped-plot submitted to simple initial and boundary conditions. To simplify the further calculations, the plot is considered to be horizontal and the gravity force sloped with regard to the vertical axis. The domain of resolution is then semi infinite on the vertical direction and infinite in the horizontal direction. After the resolution of equations, a rotation of the medium produces solutions to the initial theoretical problem (see Figure 1). 


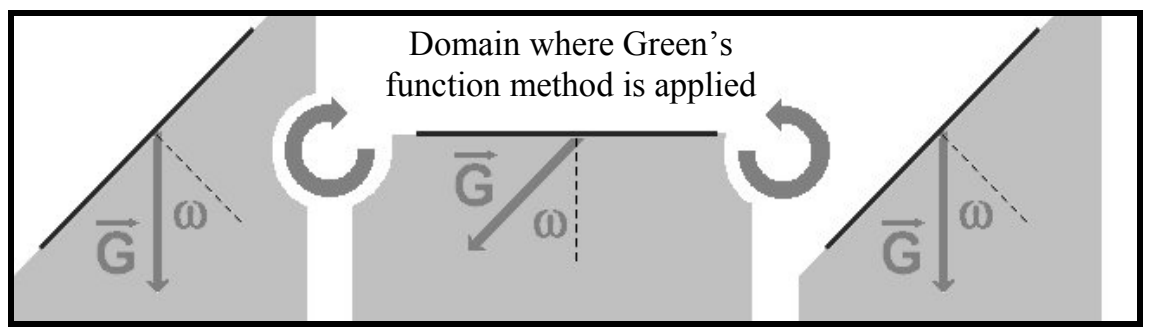

Figure 1: $\quad$ Studied domain transformations.

\subsection{The Richards' equation}

Water transfers are submitted to Richards' equation [10]. Considering the previous transformation and $\omega$ the angle between gravity force and vertical axis, this equation can be written

$$
\nabla[k \nabla(h-\sin (\omega) x-\cos (\omega) z)]=\partial_{t} \theta+S
$$

where $k$ is the hydraulic conductivity $\left(\mathrm{cm} \cdot \mathrm{h}^{-1}\right), h$ the pressure head $(\mathrm{cm}), \theta$ the water content $\left(\mathrm{cm}^{3} \cdot \mathrm{cm}^{-3}\right), z$ the vertical coordinate taken positive downward $(\mathrm{cm})$ and $S$ a sink or source term, usually the plant uptake $\left(\mathrm{h}^{-1}\right)$.

This equation is highly non-linear and it's writing has to be simplified to allow its resolution using Green's function. The following three equations allow the linearization of Richards' equation by applying the Kirchhoff transformation defined in eqn. (2) and by choosing $\theta$ and $k$ relationships suited to the problem, respectively linear soil model defined in eqn. (3) and used by Warrick [15] and Gardner model [4] defined in eqn. (4).

$$
\begin{gathered}
\phi(h)=\int_{-\infty}^{h} k(h) d h \\
\theta(h)=\theta_{R}+\frac{k(h)}{\kappa} \text { there } \kappa=\frac{k_{S}}{\left(\theta_{S}-\theta_{R}\right)} \\
k(h)=k_{S} e^{\alpha h}
\end{gathered}
$$

where $k_{S}$ is the saturated hydraulic conductivity $\left(\mathrm{cm} \cdot \mathrm{h}^{-1}\right), \alpha$ the inverse of the capillary length $\left(\mathrm{cm}^{-1}\right), \theta_{R}$ and $\theta_{S}$, the retention and saturated water content $\left(\mathrm{cm}^{3} \cdot \mathrm{cm}^{-3}\right) . \phi$ is the flux potential $\left(\mathrm{cm}^{2} \cdot \mathrm{h}^{-1}\right)$. The resulted linear PDE is then submitted to two transformations. First, dimensionless variables are introduced and a function change is used. The Richards' equation become

$$
\partial_{T} \Psi=\Delta \Psi
$$

with the following dimensionless variables 


$$
\frac{X}{x}=\frac{Z}{z}=\frac{\alpha}{2} ; \quad \frac{T}{t}=\frac{\alpha \kappa}{4} ; \quad \frac{\Phi}{\phi}=\frac{\alpha}{K_{s}}
$$

and the following function change

$$
\Psi=e^{(-X \sin \omega-Z \cos \omega+T)} \Phi
$$

the initial condition is

$$
[\Psi]_{T=0}=e^{(-X \sin \omega-Z \cos \omega)} \Phi_{i}
$$

In the context of soil water transfers in irrigation, two types of boundary conditions are considered. They are also affected by the introduction of dimensionless variables and function change. Considering $\Phi_{0}$ and $q_{0}$ the dimensionless charge and flux on the surface, the Dirichlet and Cauchy boundary conditions respectively become

$$
\begin{gathered}
{[\Psi]_{Z=0}=e^{(-X \sin \omega+T)} \Phi_{0}} \\
{\left[\partial_{Z} \Psi-(2-\cos \omega) \Psi\right]_{Z=0}=e^{(-X \sin \omega+T)} q_{0}}
\end{gathered}
$$

\subsection{The Green's function method}

Green's function method gives analytical solutions to PDE with complex boundary conditions. It involves multiplying the initial PDE by the Green's function $G$ and integrating the result. The use of Green's function is fully developed by Greenberg [5]. This function $G\left(X_{S}, Z_{S}, T_{S}\right)$ is the solution to the initial PDE submitted to an infinite pulse at the point $\left(X_{S}, Z_{S}\right)$ and time $T_{S}$ as the initial condition. Green's function depends on the type of boundary conditions considered in the PDE but is, in both cases, the linear combination of functions $G_{I D}\left(X, X_{S}, T, T_{S}\right) G_{I D}\left(Z, Z_{S}, T, T_{S}\right)$ defined in eqn. (11).

$$
G_{1 D}\left(U, U_{S}, T, T_{S}\right)=\frac{1}{\sqrt{4 \pi\left(T-T_{S}\right)}} e^{-\frac{\left(U-U_{S}\right)^{2}}{4\left(T-T_{S}\right)}}
$$

Thanks to the Green's function, the solution of the PDE considered in the eqn. (5) can be analytically written

$$
\begin{aligned}
\Psi & =\int_{0}^{\infty} \int_{-\infty}^{\infty}[G \Psi]_{T_{S}=0} d X_{S} d Z_{S} \\
& +\int_{0}^{T} \int_{-\infty}^{\infty}\left[\Psi \partial_{Z_{S}} G-G \partial_{Z_{S}} \Psi\right]_{Z_{S}=0} d X_{S} d T_{S}
\end{aligned}
$$

where the first integral accounts for the initial condition and the second for the boundary condition at the soil surface. 

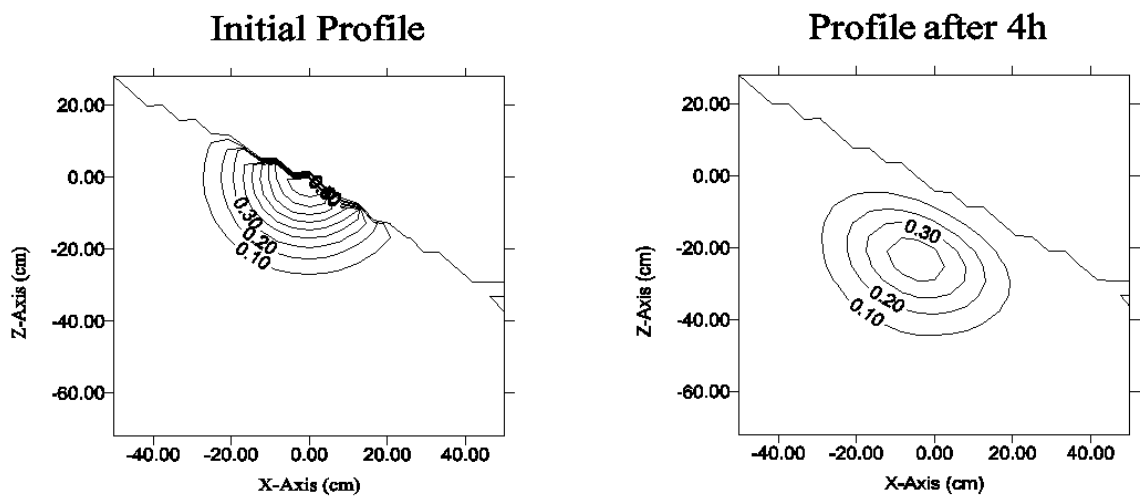

Figure 2: Dimensionless flux potential after $4 \mathrm{~h}$ simulation with a Gaussian initial condition.
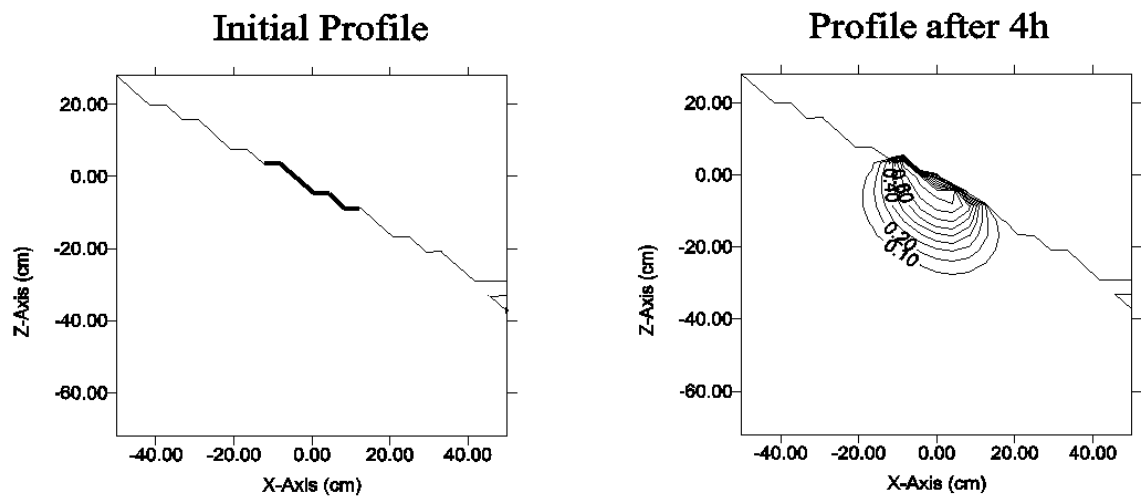

Figure 3: Dimensionless flux potential after $4 \mathrm{~h}$ simulation with a constant pressure head.

\subsection{Some results for theoretical cases}

Green's function allows the analytical writing of the solution. But explicit evaluation of eqn. (12) is only possible in particular configurations. This paragraph describes some of these configurations and gives their solutions.

Four kinds of elementary solutions are analysed in this section. The first case studied is a semi infinite medium with an initial condition $\Phi_{\mathrm{i}}(\mathrm{X}, \mathrm{Z})$, where $\Phi_{\mathrm{i}}$ $(\mathrm{X}, \mathrm{Z})$ is a Gaussian distribution. The boundary condition considered at the surface is either no pressure head, or no flux. The initial Gaussian condition is well adapted when using Green's function; the evaluation of the eqn. (12) is explicit in the case of Dirichlet boundary conditions and easy to obtain, semi analytically, in the case of Cauchy boundary conditions. The second case we analysed is a semi infinite medium with a zero initial condition and a constant pressure head or flux at the soil surface. The two following plots show the evaluations of the eqn. (12) in the case of Dirichlet boundary condition. In Figure 3 , the bold line represents the segment of the surface which is submitted to a 
constant pressure head. The same type of results can be obtained in the case of Cauchy boundary conditions.

These elementary problems can be considered as parts of more complex global problem just like analytic element method described by Strack [13]. The next section gives explanations to link these elementary bricks together in order to obtain the global solution of the more complex initial problem.

\section{Recomposition of the complete solution}

\subsection{Theory}

The previous section explains how to solve the Richards' equation in simple theoretical cases. Using these results, this section explains how to recompose the solution of a more complex problem. Let's consider the following flow problem illustrated on the left side of Figure 4.

Figure 4 represents a furrow irrigation event with a given initial water content and a given water height in the furrow. In this case, no time variable water height is considered. The geometry considered is a half furrow (due to the symmetry of the system, a half furrow vertically limited by no lateral flux boundary conditions is sufficient to represent the event). The boundary conditions on furrow surface depend on the position of the water level. Under this position, the boundary conditions are considered as variable pressure head boundary conditions, on the other part of the soil surface, the boundary conditions are considered to be no flux boundary conditions. The vertical no lateral flux boundary conditions are simulated by reproducing the symmetry of the system.
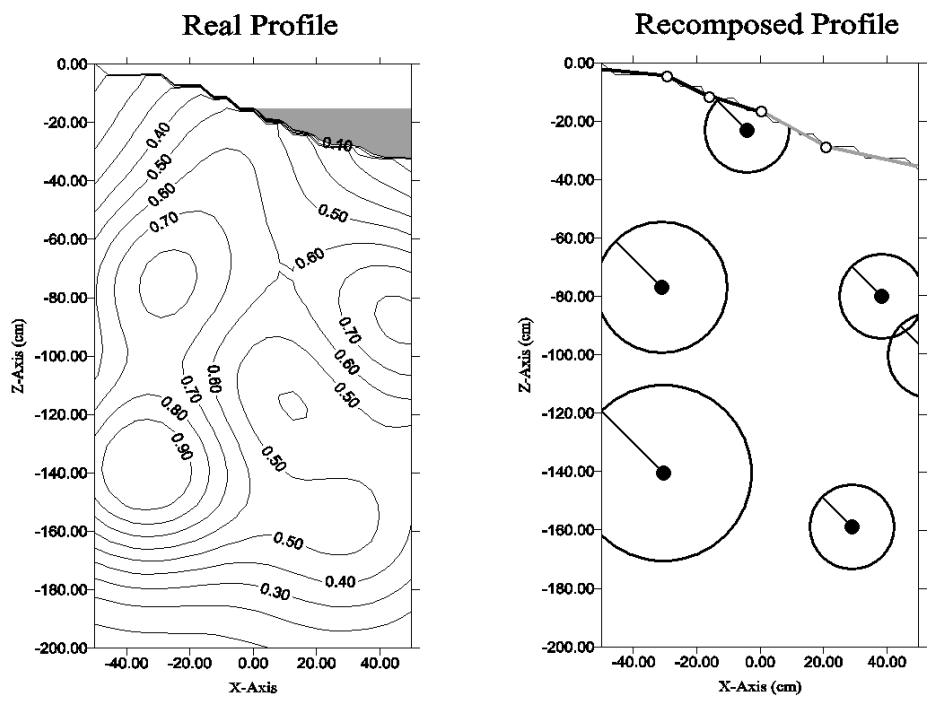

Figure 4: Recomposed initial profile to the model a complex event. 
To solve this problem, the shape of the furrow is discretized in segments with specified constant boundary conditions and the initial conditions are composed of several Gaussians (represented on the right side of Figure 14 by circles of different radius standing for different Gaussians amplitudes). The solutions to each of these elementary problems are known thanks to the study previously explained in this paper. The analytical form of the PDE solution from eqn. (12) is rewritten as a sum of these elementary problems.

$$
\begin{aligned}
\Psi & =\sum_{i} \int_{0}^{\infty} \int_{-\infty}^{\infty}\left[G \Psi_{i}\right]_{T_{S}=0} d X_{S} d Z_{S} \\
& +\sum_{j} \int_{0}^{T} \int_{\Gamma_{j}}\left[\Psi \partial_{\vec{n}_{j}} G-G \partial_{\vec{n}_{j}} \Psi\right]_{\Gamma_{j}} d \Gamma_{S} d T_{S}
\end{aligned}
$$

Where $\Psi_{i}$ are the different Gaussian distributions which make up the initial conditions and $\Gamma_{j}$ and $n_{j}$ are respectively the different segments composing the surface boundary and their normal vectors.

\subsection{The case of micro irrigation}

A first model validation concerns the case of micro irrigation practice. Modelling has been done in $2 \mathrm{D}$ context, but the transition to axisymmetric or tridirectional coordinates can be treated by Green's function with few modifications. Geometry is rectangular and boundary conditions are only Cauchy type: constant flux on a segment of the surface and no flux on the other part of the surface. The validation has been led by comparison with the numerical model Hydrus-2D [12] on an initial homogeneous wet Clay Loam soil $\left(\Delta \theta=\theta_{\mathrm{S}}-\theta_{\mathrm{I}}=0.05 \mathrm{~cm}^{3} . \mathrm{cm}^{-3}\right)$ for $4 \mathrm{~h}$ irrigation. Results are illustrated in Figure 5. Simulation performed gives satisfying results.
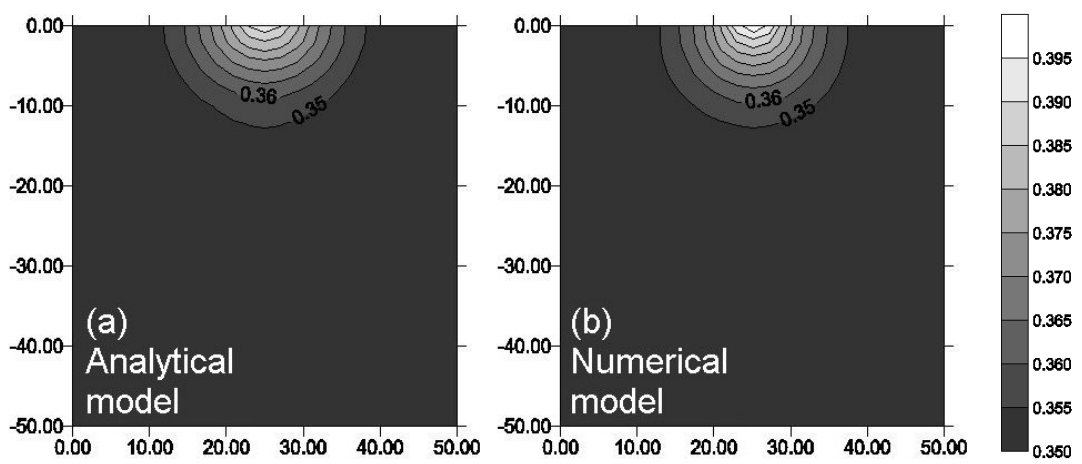

Figure 5: Comparison between analytical and numerical modelling of water content profile under micro irrigation. 


\subsection{The case of furrow irrigation}

Furrow irrigation modelling is more complex than the previous case and the reliability of the model is questionable. Validation is also carried out by comparing it with the Hydrus-2D numerical model using the same soil characteristics as employed previously. Analytical model discretizes the furrow as described in the previous section. Simulation performed concerns a $4 \mathrm{~h}$ furrow irrigation event on the homogeneous dryer soil $\left(\Delta \theta=0.14 \mathrm{~cm}^{3} . \mathrm{cm}^{-3}\right)$. Figure 6 gives the results of this comparison. Due to the linear soil assumption, the wetting front decreases from saturated water content to initial water content is lower in the simulation performed using the analytical model. The numerical modelling takes into account the relationship between soil characteristics and moisture conditions, and the wetting front is also governed by this relationship.
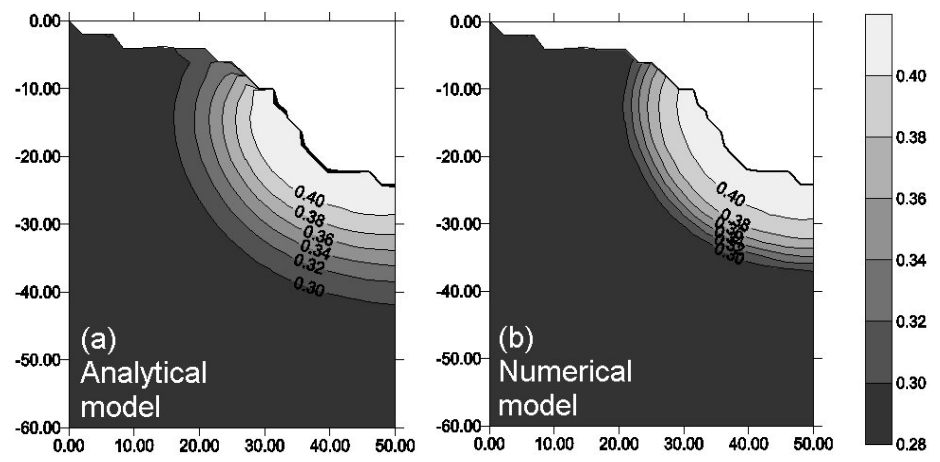

Figure 6: Comparison between analytical and numerical modelling of water content profile under furrow irrigation.

\section{Discussion and complements}

The Green's function method has been used to provide analytical solutions to the Richards' equation. Some theoretical cases provided an explicit or semi implicit evaluation of these analytical solutions and these elementary solutions can be linked together to build solutions for a more complex global problem. The advantage of this semi analytical model with regards to other analytical models is its adaptability. It's able to simulate heterogeneous initial conditions and takes into account complex boundary conditions and geometries. Concerning the numerical models, it is more operative and reduces the computing time. No notion of CFL (Courant Friedrichs Lewy) condition is introduced (imposed relationship between time and space steps for the stability of the numerical scheme), the initial conditions are easier to build for given experimental data and the number of elementary problems to solve is less than the number of cells using in numerical models.

However, this work is based on some assumptions that permit the linearization of the equations and the application of Green's function. Some of 
these assumptions, especially those of linear soil conditions, defined by the eqn. (3) can lead to difficulties in representing real water transfers in soil. In micro irrigation contexts, soil water content is generally maintained close to field capacity and its variation is low. In that case, the linear soil assumption is acceptable and its impact on results is low (see Figure 5). In the furrow irrigation context, soil moisture variations are higher. This form of irrigation often results in higher water application depth, but the irrigations events are less frequent. Consequently, the impact of the linear soil assumption is greater as shown in Figure 6.

On the way of reducing these impacts is proposed by means of an iterative process: a run of the analytical model provides an approximation of soil characteristics, based on an evaluation of PDE coefficients for a next model iteration. Another more mathematical approach to improve the linearization of water content model was proposed by Basha [1]. He introduced a perturbation solution in the original nonlinear problem.

The methods applied in this work can therefore be completed by the modelling of other phenomena that occur during a cropping season. For instance, the approach developed in this article gives the soil water content profile which is necessary for the evaluation of the nitrate transfers equation coefficients. This equation can be solved using the same principles as those used for the resolution of the Richards' equation.

\section{References}

[1] Basha, H.A., Multidimensional nonsteady infiltration with prescribed boundary conditions at the soil surface. Water resources research, 1999. 35: p. 75-84.

[2] Brisson, N., et al., STICS: a generic model for the simulation of crops and their water and nitrogen balances. I. Theory and parameterization applied to wheat and corn. Agronomie, 1998. 18(5-6): p. 311-346.

[3] Coelho, F.E. and D. Or, Applicability of analytical solutions for flow from point sources to drip irrigation management. Soil Sci. Soc. Am. J., 1997. 61: p. 1331-1341.

[4] Gardner, W.R. and M.S. Mayhugh, Solution and tests of the diffusion equation for the movement of water in soil. Soil Science Society of Am. Proc., 1958. 22: p. 197-201.

[5] Greenberg, M.D., Application of Green's functions in science and engineering. 1971, Englewood Cliffs, N.J.: Prentice-Hall.

[6] Jones, C.A. and J.R. Kinity, CERES-MAIZE, a Simulation Model of Maize Growth and Development. 1986: Texas A.M. University Press.

[7] Mailhol, J.-C., P. Ruelle, and I. Nemeth, Impact of fertilisation practices on nitrogen leaching under irrigation. Irrigation Science, 2001. 20: p. 139147.

[8] Philip, J.R., Linearized unsteady multidimensional infiltration. Water resources research, 1986. 22(12): p. 1717-1727. 
[9] Popova, Z., et al. Lysimeter study on ground water degradation due to different fertilisation and irrigation management. in ICID-ICWRM in the 21st Century. 2000. Budapest, Hungary.

[10] Richards, L.A., Capillary condiction of liquids through porous medium. Physics, 1931. 1: p. 318-333.

[11] Schmitz, G.H., Transient infiltration from cavities - I : theory. Journal of irrigation and drainage engineering, 1993. 119(3): p. 443-457.

[12] Simunek, J., M. Sejna, and M.T. Van Genuchten, The HYDRUS-1D and HYDRUS-2D codes for estimating unsaturated soil hydraulic and solutes transport parameters. Agron. Abstr., 1999. 357.

[13] Strack, O.D.L., Groundwater Mechanics. 1989, Englewood Cliffs, N.J.: Prentice-Hall.

[14] Van Genuchten, M.T., A numerical model for water and solute movement in and below the root zone. Research Report. 1987, U.S. Salinity laboratory, USDA, ARS,: Riverside, California.

[15] Warrick, A.W., Time-depend linearized infiltration. I. Point sources. Soil science society of Am. Proc., 1974. 38(12): p. 383-386.

[16] Wöhling, T., G.H. Schmitz, and J.-C. Mailhol, Modelling 2D-infiltration from irrigation furrow. Analysis of analytical and numerical approaches. Journal of Irrigation and Drainage Engineering, 2004. 130(4): p. 296-303. 